\title{
Prevalence of depression symptoms and associated sociodemographic and clinical correlates among Syrian refugees in Lebanon
}

Hady Naal ${ }^{1}$, Dana Nabulsi ${ }^{1}$, Nour El Arnaout ${ }^{1}$, Lina Abdouni ${ }^{1}$, Hani Dimassi ${ }^{2}$, Ranime Harb ${ }^{2}$ and Shadi Saleh ${ }^{1,3^{*}}$

\begin{abstract}
Background: Since the outbreak of the Syrian war in 2011, close to 6 million Syrian refugees have escaped to Syria's neighbouring countries, including Lebanon. Evidence suggests rising levels of mental health disorders among Syrian refugee populations. Yet, to the best of our knowledge, large-scale studies addressing the mental health of adult Syrian refugees in Lebanon are lacking. We examined the prevalence of depression symptoms, which represent a common and debilitating mental health disorder among Syrian refugee populations in Lebanon, along with their sociodemographic and clinical correlates.

Methods: A cross-sectional survey design was conducted as part of a collaborative project-"Sijilli"- led by the Global Health Institute at the American University of Beirut (Beirut, Lebanon) across 4 informal tented settlements for refugees (Beirut, Bekaa, North, South) in Lebanon among adult Syrian refugees $(\geq 18)$, over a period extending from 2018 to 2020. The survey inquired about participants' sociodemographic and clinical characteristics, and screened participants for symptoms of depression through sequential methodology using the Patient Health Questionnaire (PHQ-2 and PHQ-9).

Results: A total of 3255 adult Syrian refugees were enrolled in the study. Of those refugees, $46.73 \%(n=1521)$ screened positive on the PHQ-2 and were therefore eligible to complete the PHQ-9. In the entire sample $(n=3255)$, the prevalence of moderate to severe depression symptoms (PHQ-2 $\geq 2$ and then PHQ-9 $\geq 10)$ was $22 \%(n=706)$. Further analyses indicate that being $\geq 45$ years of age (OR 1.61, 95\% Cl 1.13-2.30), a woman (OR 1.34, 95\% Cl 1.061.70), widowed (OR 2.88, 95\% Cl 1.31-6.32), reporting a neurological (OR 1.73, 95\% Cl 1.15-2.60) or a mental health condition (OR 3.98, 95\% Cl 1.76-8.97) are major risk factors for depression.

Conclusion: Our study suggests that an estimated one in four Syrian refugees in Lebanon shows moderate to severe depression symptoms, and our findings have important public health and clinical implications on refugee health. There is a need to enhance screening efforts, to improve access and referral to mental health services, and to improve post-migration factors among Syrian refugees in Lebanon.
\end{abstract}

Keywords: Syrian refugees, Informal tented settlements, Sijilli, Mental health, Depression, Lebanon

\footnotetext{
* Correspondence: ss117@aub.edu.lb

${ }^{1}$ Global Health Institute, American University of Beirut, Beirut, Lebanon

${ }^{3}$ Faculty of Health Sciences, American University of Beirut, Beirut, Lebanon

Full list of author information is available at the end of the article
}

(C) The Author(s). 2021 Open Access This article is licensed under a Creative Commons Attribution 4.0 International License, which permits use, sharing, adaptation, distribution and reproduction in any medium or format, as long as you give appropriate credit to the original author(s) and the source, provide a link to the Creative Commons licence, and indicate if changes were made. The images or other third party material in this article are included in the article's Creative Commons. licence, unless indicated otherwise in a credit line to the material. If material is not included in the article's Creative Commons licence and your intended use is not permitted by statutory regulation or exceeds the permitted use, you will need to obtain permission directly from the copyright holder. To view a copy of this licence, visit http://creativecommons.org/licenses/by/4.0/ The Creative Commons Public Domain Dedication waiver (http://creativecommons.org/publicdomain/zero/1.0/) applies to the data made available in this article, unless otherwise stated in a credit line to the data. 


\section{Background}

The Syrian crisis has been widely described as one of the largest refugee crises of recent times [1,2]. Close to 6 million Syrian refugees have fled to Syria's neighbouring countries, namely Lebanon, Jordan, Turkey, Egypt, and Iraq [3]. Lebanon currently hosts over 1.5 million Syrian refugees, a number equivalent to $25 \%$ of its population [4], rendering it the country with the largest number of refugees per capita worldwide [5]. The massive influx of Syrian refugees to Lebanon, coupled with their increased demand for healthcare services, has significantly strained the country's already fragile healthcare system, and has hindered its ability to cater to their health needs $[6,7]$.

Having escaped from conflict settings, refugees often experience a multitude of stressors such as traumatic events, multiple forms of losses, discrimination, and acculturation difficulties among others during their journey of displacement [1]. They have therefore significantly higher odds of developing mental health disorders compared to the general population [1]. Many refugees are survivors of exploitation, torture, and sexual and gender-based violence, which further exacerbate their vulnerability to health conditions [1]. That said, they are less likely to receive mental health services because of social stigma, language and cultural barriers, imbalanced power dynamics with service providers, limited access to services, and low mental health literacy, including lack of perceived need [1, 8-10]. Recent evidence indicates that mental health is one of the most pressing health needs among Syrian refugees in Lebanon and neighbouring countries [11].

Major Depression Disorder (MDD), Post-Traumatic Stress Disorder (PTSD), and other anxiety disorders have been reported as the most common mental health disorders among Syrian refugees [12, 13], and they tend to be comorbid conditions. In the general population, MDD in particular, is the third leading cause of years lived with disability (YLDs) [14], and is considered a strong risk factor for suicide $[15,16]$. Therefore, depression warrants special attention among this population due to its long-term implications that may impair social, individual, and vocational functioning, factors that are essential for survival, productivity, and resettlement [17, 18]. In one meta-analysis that included 24,051 refugees from multiple nationalities pooled from international studies, $44 \%$ were found to have symptoms of depression [19]. Despite certain limitations of that review, such as heterogeneity of the included samples, this finding mirrors others in the literature addressing Syrian refugees in Arab settings [20-23]. Furthermore, previous reports from the literature assessing prevalence rates of depression among Syrian refugees in developed and developing countries have shown disparities in the findings. Depression prevalence rates were reportedly lower in developed countries compared to developing countries, which could be attributed to the limited capacities of the latter to cope with the needs of these vulnerable populations. In Germany, depression was detected in close to $14.5 \%$ of a Syrian refugee sample [13], as opposed to $37.4 \%$ in Turkey [12], and 43\% in Lebanon [20].

Previous research suggests that despite the harsh conditions that Syrian refugees often experience, some key post-displacement variables may act as protective factors, may buffer the severity and incidence of mental health conditions, and may contribute to posttraumatic growth [13, 24]. For instance, obtaining a visa or residence permission, residing in acceptable living conditions, receiving financial support, and having access to social and healthcare services, among others, have contributed to better mental health outcomes [13]. Rightfully so, findings have confirmed that besides the existing acute and chronic stressors, the mental health of refugees largely depends on the social, economic, and cultural environments associated with their pre and post-displacement experiences [25]. However, the status of Syrian refugees in Lebanon is far from being ideal, with potential protective factors being compromised and minimally available [26]. As an example, Syrian refugees have restricted rights in Lebanon, which limit their access to proper healthcare, education, and employment opportunities, due to the lack of a clearly defined legal and administrative framework under which they can operate [26, 27]. Such systemic precariousness excludes potential opportunities for long-term integration, and places Syrian refugees in Lebanon at increased risk of developing mental health problems [28, 29]. In addition to that, Syrian refugees lack basic needs such as food, water, and shelter. Their acculturation is also compromised, as they tend to experience discrimination resulting from the strained Lebanese-Syrian relations due to host community fatigue with their protracted presence, largely due to job competition and exhaustion of resources and services [30]. This discrimination is also manifested as part of the larger socio-political climate in which continuous pressures are being exerted on Syrian refugees that threaten their physical, financial, and social security $[26,31,32]$.

Finally, the lack of sustained funding for mental health services, the fragmented mental healthcare system, and the scarcity of research make it extremely difficult to understand and respond to the psychosocial needs of refugees. Syrian refugees, being a high-risk group, have unique psychosocial needs that should be clearly addressed by mental health workers, based on evidencedriven and culturally-sensitive findings. That said, concerted efforts have been made by the National Mental Health Program (NMHP) at the Ministry of Public Health $(\mathrm{MoPH})$ in Lebanon in collaboration with other 
healthcare and humanitarian actors to address this problem $[6,7,33,34]$. Since the inception of the Mental Health and Substance Use Strategy for Lebanon 20152020 , the MoPH has worked on integrating mental health services into the primary healthcare centres (PHCs) by training healthcare workers on the Mental Health Gap Action Program (mhGAP) to enhance access to mental health care [35]. In addition, the MoPH established the Mental Health and Psychosocial Support Task Force (MHPSS-TF) in collaboration with the World Health Organization (WHO) and the UNICEF to coordinate the work of over 62 actors on the mental health and psychosocial support within the Syrian crisis response in Lebanon [7]. This has increased the efficiency and effectiveness of efforts targeting this problem.

In this context and despite the established initiatives, to our knowledge, there are limited large-scale studies on the prevalence of depression and its correlates among adult Syrian refugees in the Middle East. In fact, despite the urgency of the crisis, according to a recent systematic review, only six studies emanating from a conflictaffected low-to middle-income country were conducted in the Middle East to assess the prevalence rates of mental health disorders among refugees [36]. Clearly, there is an immense need for further research on the mental health of Syrian refugees in this region to better understand the associated risk factors, and to support the development and implementation of global mental health policies addressing this population [36].

The present study aims to examine the prevalence of depression symptoms and their sociodemographic and clinical correlates among Syrian refugees in Lebanon. In doing so, we provide researchers, policy makers, and practitioners with a comprehensive understanding of depression among migrating populations, which would constitute a foundational base for future interventions and related programs and policies.

\section{Methods}

\section{Design \& Population}

Our study is a secondary analysis of de-identified data from the "Sijilli" (Electronic Health Records (EHR) for Refugees) database [37]. The Sijilli database includes data on 10,082 Syrian refugees in Lebanon, collected between July 2018 and January 2020 through primary field-based data collection conducted by the Global Health Institute at the American University of Beirut in partnership with Epic Systems Corporation. Data collection took place in different informal tented settlements for refugees across Lebanon, covering the Bekaa, North Lebanon, Beirut/Mount Lebanon, and South Lebanon areas. The sample size in each of these locations was proportionate to the overall Syrian refugee population residing in the latter based on UNHCR data [38] and includes 3565 refugee records (35.4\%) from Bekaa, 2657 refugee records (26.4\%) from North Lebanon, 2146 refugee records $(21.3 \%)$ from Beirut, and 1714 refugee records (17.0\%) from South Lebanon. The Sijilli database includes records of Syrian refugees of all ages, and covers 7 sections (see Additional File 1): sociodemographic information (e.g. age, gender, Syrian governorate of origin, location of the settlement, and year of migration to Lebanon), health behaviours (e.g smoking, alcohol drinking, and physical exercise), medical and surgical history, Obstetrics and Gynaecology (OBGYN) conditions, medication use, vaccination history, and mental health screening. The mental health screening was completed through the Patient Health Questionnaire-9 (PHQ-9). Any mental health disorder reported by refugees was noted right after PHQ-9 administration. In this study, we only analysed the data of adult Syrian refugees who were 18 years of age or above $(n=3255)$.

\section{Measures}

From the collected data, we examined sociodemographic variables of age, gender, marital status, country of origin, time of arrival to Lebanon, location of settlement, and current occupation, and clinical variables of tobacco and alcohol use, medical conditions such as diabetes, cardiovascular diseases (CVD), neurological conditions, coronary artery diseases, use of psychiatric medication, and mental health conditions. Symptoms of depression were assessed in two phases using sequential methodology, which included administering the PHQ-2 followed by the PHQ-9 for those who screen positive on the former. This method has been widely used and is especially recommended for efficient data collection in conflict settings such as informal tented settlements [39].

The PHQ-9 includes 9 items rated on 4-point Likert ranging from 0 to 3 , and yields a total score ranging from 0 to 27. The PHQ-9 score may be used as a categorical outcome of no, mild, moderate, moderately severe, and severe symptoms of depression, or may be used as a continuous score [40]. This measure screens for symptoms of depression experienced within the last 2 weeks and has previously shown evidence of validity, reliability, and unidimensionality [41]. In the present study, the PHQ-9 has shown very good reliability, with an alpha coefficient of 0.856 .

\section{Analysis}

All collected data were managed and analysed using SPSS v26. Descriptive statistics for sample characteristics were computed for adults using frequencies and percentages for categorical data. Internal consistency of the PHQ-9 was testing using Cronbach alpha. As recommended by a previous study [39], participants who 
screened positive on the PHQ-2 (received a score of 2 or above) were selected for further analysis in the regression model. PHQ-9 score was categorized into no symptoms (0-4), mild (5-9), moderate (10-14), moderately severe (15-19), and severe (20-27). Positive PHQ-9 was represented by a score of 10 or higher, as per previous studies in the literature [17]. PHQ-9 categories and average scores, as well as individual PHQ-9 items were summarized and reported as frequencies and percentages. Univariate and multiple logistic regression models were computed to determine the association of the PHQ-9 categories (mild and lower, score of $0-9$; vs moderate to severe, score of 10 or higher) with the sample characteristics. Coefficients and standard errors were exponentiated to produce odds ratios and 95\% confidence intervals. All analyses were run at the 0.05 statistical significance level.

\section{Results}

\section{Participants}

A total of $n=3255$ adult Syrian refugees were included in this study, and their characteristics are presented in Table 1 . The mean age was 36.53 with a standard deviation of 13.87. Most of the participants were women (67.1\%), married (78.4\%), had arrived to Lebanon between 2012 and 2015 (62.6\%), originating from Hama governorate in Syria (18.9\%), currently residing in the Bekaa governorate in Lebanon (32.6\%), and unemployed (74.6\%). The majority of participants reported never using tobacco (67.6\%) and alcohol (99.5\%), and only $1.2 \%$ reported using psychiatric medication such as antidepressants, antipsychotics, and anxiolytics. The most commonly reported medical condition was hypertension $(10 \%)$, and the presence of any mental health condition was reported among only $2.1 \%$ of the study population.

\section{Prevalence and distribution of depression symptoms}

Of those $n=3255$ adults, almost a quarter $(n=706)$ screened positive on the PHQ-2 (scored $\geq 2$ ) and then on the PHQ-9 (scored $\geq 10$ ), indicating a $22 \%$ prevalence of moderate to severe depression symptoms in the total population $(n=3255)$. Less than half of the sample 46.7\% $(n=1521)$ completed the full PHQ-9 and were subsequently included in the regression analysis. The remaining half $(n=1734)$ were excluded from the regression analysis due to screening negative on the PHQ-2 (see Fig. 1). More than half $(53.6 \%)$ of those who completed the PHQ-9 showed no or mild depression symptom severity, however, the rest (46.4\%) showed moderate to severe symptom severity (see Table 2). The highest reported symptom severity was for "feeling little interest and pleasure in doing things that used to be enjoyable", "feeling down and depressed on most days", and "having little energy to complete daily tasks". Whereas the least reported symptoms were for "moving or speaking very slowly", and "having suicidal thoughts".

\section{Sociodemographic and clinical correlates of depression symptoms}

Based on the results of the first regression model at the bivariate level, being over 45 (OR 1.74, 95\% CI 1.252.14), a woman (OR 1.38, 95\% CI 1.00-1.74), divorced/ separated (OR 3.27, 95\% CI 1.51-7.08), on psychiatric medication (OR 2.73, 95\% CI 1.04-7.14), having hypertension (OR 1.36, 95\% CI 1.00-1.84) or any nervoussystem related disorder (OR 1.76, 95\% CI 1.78-2.63), or mental health condition (OR 3.70, 95\% CI 1.67-8.26) significantly increased the odds for showing more severe symptoms of depression (see Table 3). On the other hand, informal tented settlement location, current occupation, tobacco and alcohol use, and having diabetes or CVDs showed no statistically significant association with symptoms of depression.

At the multivariable level of analysis, comparable results were obtained, whereby being $\geq 45$ of age (OR 1.61, 95\% CI 1.13-2.30), a woman (OR 1.34, 95\% CI 1.061.70 ), widowed (OR 2.88, 95\% CI 1.31-6.32), reporting a neurological disorder (OR 1.73, 95\% CI 1.15-2.60) or a mental health condition (OR 3.98, 95\% CI 1.76-8.97) significantly increased the odds of screening positive for moderate to severe depression symptoms (see Table 4). However, unlike the bivariate model, being on psychiatric medication and presenting with hypertension were not significantly associated with the increased odds of depression severity symptoms.

\section{Discussion}

In the present study, we report the prevalence of depression symptoms, which represent one of the most common and debilitating mental health disorders among Syrian refugees, and we explore their sociodemographic and clinical correlates. The main strength of this study is that to our knowledge, it is one of the very few regional, and the only national large-scale study addressing this issue among a representative sample of adult Syrian refugees in Lebanon.

As expected, the prevalence of depression symptoms among the study population was high, with an estimated one in four refugees meeting criteria for moderate to severe depression symptoms. Positive PHQ-9 screening and consequently, moderate to severe depression symptoms, was detected in $25 \%$ of the study population, which is considerably higher than depression rates (9.9\%) previously reported among the general population in Lebanon [42]. Our findings contrast a previous study that used the same depression measure on Syrian refugees in Germany, and in which only $14 \%$ of their sample screened positive for moderate to severe depression 
Table 1 Characteristics of the present sample $(N=3255)$

\begin{tabular}{l}
\hline \\
\hline Gender \\
Female \\
Male \\
Age (mean \& SD) \\
18-25 \\
26-35 \\
36-45 \\
$>45$
\end{tabular}

Marital Status

Married

Single

Divorced

Widowed

Separated

City of Origin

Hama

Aleppo

Raqqa

Idlib

Homs

Deir ez-Zor

Others

Time of Arrival to Lebanon

$$
\begin{aligned}
& <2011 \\
& 2012-2015 \\
& 2016-2020
\end{aligned}
$$

\section{Location}

Beirut

Bekaa

North Lebanon

South Lebanon

\section{Current Occupation}

Unemployed

Employed

\section{Exercise}

Yes

No

\section{Tobacco Use}

No use
Ex-user
Current
Hookah
Cigar

Total

N (\%)

2184

1071

$36.53(13.87)$

825

878

772

780

2553

552

32

106

12

616

606

515

498

456

299

262

616

2039

600

670

1062

942

581

2420

826

611

2328

2109

91

920

171

46
Table 1 Characteristics of the present sample $(N=3255)$

(Continued)

\begin{tabular}{lll}
\hline & Total & \\
\cline { 2 - 3 } & N (\%) & \\
\hline \multicolumn{1}{c}{ Cigarettes } & 797 & $24.50 \%$ \\
Alcohol Use & & \\
Yes & 17 & $0.50 \%$ \\
No & 2992 & $99.50 \%$ \\
Medication Use (Psychiatric) & & \\
Antidepressant & 28 & $0.9 \%$ \\
Antipsychotic & 9 & $0.3 \%$ \\
Anxiolytic & 7 & $0.2 \%$ \\
Medical Conditions & & \\
Hypertension & 326 & $10.0 \%$ \\
Neurological disorders & 196 & $6.0 \%$ \\
Diabetes & 185 & $5.7 \%$ \\
Cardiovascular Disease & 175 & $5.4 \%$ \\
Coronary Artery Disease & 62 & $1.9 \%$ \\
Mental Health Conditions & & $1.23 \%$ \\
Any Mental Health Condition & 40 & \\
\hline
\end{tabular}

symptoms [13]. One of the main findings of that study was that post-migration conditions and future positive prospects in host countries may be protective against mental health disorders among these populations [13]. Indeed, this could explain the discrepancy between their findings and ours, considering that post-migration factors in Lebanon are poor and may ultimately present important risk factors instead of being protective against mental health disorders [26].

In Lebanon, many post-migration variables present important obstacles towards adequate health and survival. For example, the high prevalence of depression symptoms could be attributed to numerous external factors beyond their exposure to traumatic events, such as the difficult conditions in which Syrian refugees live, the limited opportunities for development, and the many challenges associated with their social integration and acculturation [26]. Additionally, the constant internal and regional socioeconomic and political conflicts promote little hope for refugees to settle in a stable context unless they travel to more developed countries, which is a solution Syrian refugees commonly request to overcome their documented adverse living conditions [26]. The existing economic difficulties in Lebanon, which are now exponentially compounded by the fall of Lebanese pound [43], and by the colossal explosion that devastated the capital Beirut in August 2020 [44], may place Syrian refugees under further instability and vulnerability. With that said, the situation is currently expected to be worse in terms of mental health outcomes, 


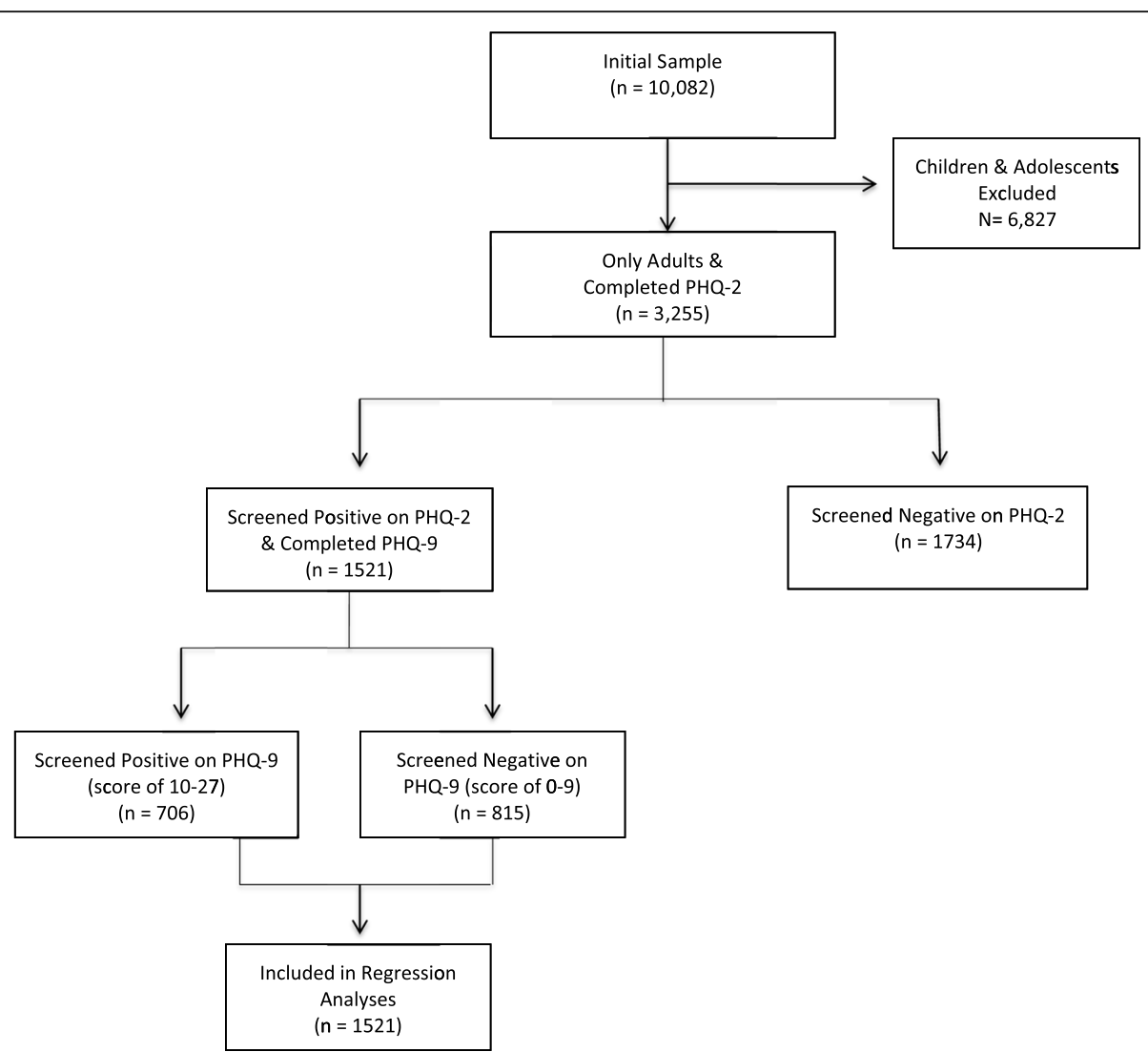

Fig. 1 Flowchart for participant selection. This flowchart showcases the process by which recruited participants were selected from inclusion in the study analysis

considering the COVID-19 pandemic, which has restricted mobility, tremendously challenged the attainment of basic survival needs, induced added stress, and further limited opportunities for work and social interactions [45].

In response to the Syrian crisis, the $\mathrm{MoPH}$ in collaboration with the Ministry of Social Affairs and with local and international non-governmental organizations (NGOs) have been providing free-of-charge primary healthcare services, including mental health services, for UNHCR-registered Syrian refugees. Yet, due to limited financial capacities, these efforts have been reportedly unable to meet the increasing needs of these vulnerable populations [46, 47]. The situation is even worse for unregistered refugees who have restricted capacities to receive the appropriate healthcare support [47]. Despite these efforts, symptoms of depression remain high, as indicated by our findings. Although mental health services and psychosocial interventions may induce relief of depression severity over the short-term, long-term improvements may require complementary macro-level changes in the living conditions of refugees, their legal status, and the need to foster positive future prospects.
It is also possible that mental health services are not reaching enough refugees. Notwithstanding the value of the provided services, Syrian refugees cite many barriers to seeking mental health services in Lebanon, including lack of trust in and limited knowledge of available services, limited mental health literacy and perceived need for treatment, lack of services especially in rural areas, associated difficulties in commuting, financial barriers and lack of mental health coverage, and social stigma which may impede refugees seeking healthcare fearing of shame and discrimination [46]. Furthermore, due to pervasive cultural beliefs, Syrian refugees tend to seek religious healers as a first line of treatment for mental illness given their perceived cultural appropriateness and their reduced association with social stigma when compared to mental health professionals [48].

Despite high symptoms of depression in our sample, our findings are favourable in comparison to the last study that evaluated the prevalence of depression among adult Syrian refugees in Lebanon 5 years ago, in which a depression prevalence rate of $43.9 \%$ was reported [20]. Although some important limitations may prohibit this comparison, such as their reliance on a clinical diagnosis 
Table 2 Distribution of depression symptoms $N=1521$

\begin{tabular}{|c|c|c|c|c|c|}
\hline & $\mathbf{N}$ & $\%$ & (Continued) & & \\
\hline Depression Symptoms & & & & $\mathrm{N}$ & $\%$ \\
\hline No symptoms (0-4) & 201 & 13.2 & Nearly everyday & 145 & 9.5 \\
\hline Mild (5-9) & 614 & 40.4 & PHQ8: Move/speak slowly & & \\
\hline Moderate (10-14) & 372 & 24.5 & Not at all & 805 & 52.9 \\
\hline Moderately Severe e(15-19) & 210 & 13.8 & Several days & 422 & 27.7 \\
\hline Severe (20-27) & 124 & 8.2 & More than half of days & 199 & 13.1 \\
\hline Mean (SD) & $10.4(5.7)$ & & Nearly everyday & 81 & 5.3 \\
\hline Depression Screening & & & PHQ9: Better off dead & & \\
\hline Mild and lower (0-9) & 815 & 53.6 & Not at all & 932 & 61.3 \\
\hline Moderate to Severe (10-27) & 706 & 46.4 & Several days & 320 & 21.0 \\
\hline PHQ1: Interest/pleasure & & & More than half of days & 155 & 10.2 \\
\hline Not at all & 38 & 2.5 & Nearly everyday & 104 & 6.8 \\
\hline Several days & 796 & 52.3 & PHQ10 Effect work/people & & \\
\hline More than half of days & 395 & 26.0 & Not difficult at all & 485 & 31.9 \\
\hline Nearly everyday & 285 & 18.7 & Somewhat difficult & 621 & 40.8 \\
\hline PHQ2: Down, depressed & & & Very difficult & 255 & 16.8 \\
\hline Not at all & 14 & .9 & Extremely difficult & 124 & 8.2 \\
\hline
\end{tabular}

Table 2 Distribution of depression symptoms $N=1521$

(Continued)
Nearly everyday

PHQ3: Sleep

Not at all

Several days

More than half of days

Nearly everyday

PHQ4: Energy

Not at all

Several days

More than half of days

Nearly everyday

\section{PHQ5: Appetite}

Not at all

Several days

More than half of days

Nearly everyday

PHQ6: Feeling bad/failure

Not at all

Several days

More than half of days

Nearly everyday

PHQ7: Concentrating

Not at all

Several days

More than half of days as opposed to using a screening instrument, and their smaller sample size $(n=310)$ [20], our findings may point towards an overall improvement. However, this could also be a result of the different sample characteristics, considering that Naja et al. [20] study represented refugees seeking social and healthcare services from two non-governmental organizations, whereas ours included a randomly selected representative sample of participants from the general population of Syrian refugees across Lebanon; under these different contexts, our sample would be expected to score lower on depression.

In terms of its correlates, our findings show that age, gender, and marital status are strongly associated with an increased risk for depression. We found that older age is associated with higher risk of depression, which contrasts previous findings showing an inverse relationship [13], or no correlation [17, 20] between these variables. In specific, individuals over 45 years of age are at highest risk of developing depression, and this may be a result of several contributing factors. From a social perspective, older individuals in the Arab region are regarded as pillars of their families, and they tend to hold leadership roles in their communities $[49,50]$. Previous research suggests that as a result of the war, this community role may be disrupted, impacting familial connectedness and social ties, and bringing along feelings of isolation and inadequacy [49]. From a clinical perspective, older individuals often have pre-existing and chronic conditions which may warrant further medical attention. In this regard, unmet healthcare needs due to the limited resources in these communities may further 
Table 3 Sociodemographic and clinical correlates of depression symptoms at the bivariate level $(n=1521)$

\begin{tabular}{|c|c|c|c|c|c|c|c|}
\hline & $\mathrm{N}$ & $\%$ & $p$-value & Odds ratio & Lower L & Upper L & $\begin{array}{l}\text { ROC } \\
\text { area }(95 \% \mathrm{Cl})\end{array}$ \\
\hline \multicolumn{8}{|l|}{ Age } \\
\hline $18-25$ (ref) & 89 & $39.0 \%$ & & Ref & & & \\
\hline $26-35$ & 197 & $46.9 \%$ & & 1.380 & .994 & 1.915 & \\
\hline $36-45$ & 190 & $43.5 \%$ & & 1.201 & .867 & 1.665 & \\
\hline$>45$ & 230 & $52.8 \%$ & .004 & 1.744 & 1.259 & 2.415 & $.55(.52-.58)$ \\
\hline \multicolumn{8}{|l|}{ Gender } \\
\hline Male & 169 & $40.6 \%$ & & ref & & & \\
\hline Female & 537 & $48.6 \%$ & .006 & 1.382 & 1.100 & 1.736 & $.53(.50-.56)$ \\
\hline \multicolumn{8}{|l|}{ Marital Status } \\
\hline Married & 556 & $44.9 \%$ & & ref & & & \\
\hline Single & 85 & $47.5 \%$ & & 1.108 & .809 & 1.516 & \\
\hline Widowed & 41 & $56.9 \%$ & & 1.620 & 1.003 & 2.617 & \\
\hline Divorced/separated & 24 & $68.6 \%$ & $<.001$ & 3.266 & 1.506 & 7.084 & $.53(.50-.56)$ \\
\hline Period of Stay (continuous) & & & .947 & 1.002 & .954 & 1.051 & $.50(.47-.53)$ \\
\hline \multicolumn{8}{|l|}{ Location } \\
\hline Beirut & 128 & $43.0 \%$ & & ref & & & \\
\hline North & 187 & $46.3 \%$ & & 1.145 & .847 & 1.547 & \\
\hline South & 94 & $46.3 \%$ & & 1.145 & .800 & 1.640 & \\
\hline Bekaa & 297 & $48.2 \%$ & .524 & 1.237 & .936 & 1.634 & $.52(.49-.55)$ \\
\hline \multicolumn{8}{|l|}{ Current Occupation } \\
\hline Employed & 161 & $44.6 \%$ & & ref & & & \\
\hline Not Employed & 542 & $47.0 \%$ & .431 & .909 & .717 & 1.153 & $.51(.48-.54)$ \\
\hline \multicolumn{8}{|l|}{ Tobacco Use } \\
\hline Never User & 455 & $46.6 \%$ & & ref & & & \\
\hline Current & 234 & $46.9 \%$ & .906 & 1.013 & .816 & 1.257 & $.50(.47-.53)$ \\
\hline \multicolumn{8}{|l|}{ Alcohol Use } \\
\hline No & 675 & $47.3 \%$ & & ref & & & \\
\hline Yes & 3 & $42.9 \%$ & .814 & .836 & .186 & 3.747 & $.50(.47-.53)$ \\
\hline \multicolumn{8}{|l|}{ Psychiatry Medication Use } \\
\hline No & 692 & $46.1 \%$ & & ref & & & \\
\hline Yes & 14 & $70.0 \%$ & .033 & 2.728 & 1.043 & 7.137 & $.51(.48-.54)$ \\
\hline \multicolumn{8}{|l|}{ Medical Conditions } \\
\hline Diabetes No & 648 & $45.9 \%$ & & & & & \\
\hline Diabetes Yes & 58 & $53.7 \%$ & .115 & 1.369 & .925 & 2.028 & $.51(.48-.54)$ \\
\hline Hypertensive No & 604 & $45.4 \%$ & & & & & \\
\hline Hypertensive Yes & 102 & $53.1 \%$ & .046 & 1.360 & 1.004 & 1.842 & $.52(.49-.55)$ \\
\hline Cardiovascular Disease No & 656 & $45.9 \%$ & & & & & \\
\hline Cardiovascular Disease Yes & 50 & $53.8 \%$ & .143 & 1.368 & .898 & 2.084 & $.51(.48-.54)$ \\
\hline Nervous Disorder No & 643 & $45.4 \%$ & & & & & \\
\hline Nervous Disorder Yes & 63 & $59.4 \%$ & .005 & 1.759 & 1.177 & 2.628 & $.52(.49-.55)$ \\
\hline Mental Health No & 681 & $45.8 \%$ & & & & & \\
\hline Mental Health Yes & 25 & $75.8 \%$ & $<.001$ & 3.703 & 1.660 & 8.263 & $.51(.48-.54)$ \\
\hline
\end{tabular}

Note: the dependent variable is the dichotomized PHQ-9 (mild and lower VS moderate to severe) 
Table 4 Sociodemographic and clinical correlates of depression symptoms at the multivariable level $(N=1521)$

\begin{tabular}{|c|c|c|c|c|c|c|c|}
\hline Risk factor & B & SE & $p$-value & Odds ratio & Lower L & Upper L & ROC $(95 \% \mathrm{Cl})$ \\
\hline Multivariable Logistic Regression Model & & & & & & & $.60(.57-.63)$ \\
\hline \multicolumn{8}{|l|}{ Age groups } \\
\hline \multicolumn{8}{|l|}{$18-25$ (ref) } \\
\hline $26-35$ & .316 & .172 & .066 & 1.371 & .979 & 1.921 & \\
\hline $36-45$ & .162 & .172 & .345 & 1.176 & .840 & 1.648 & \\
\hline$>45$ & .476 & .181 & .009 & 1.610 & 1.128 & 2.296 & \\
\hline \multicolumn{8}{|l|}{ Gender } \\
\hline \multicolumn{8}{|l|}{ Male (ref) } \\
\hline Female & .293 & .121 & .015 & 1.340 & 1.058 & 1.698 & \\
\hline \multicolumn{8}{|l|}{ Marital Status } \\
\hline \multicolumn{8}{|l|}{ Married (ref) } \\
\hline Single & .194 & .166 & .243 & 1.214 & .877 & 1.681 & \\
\hline Widowed & .299 & .254 & .008 & 2.883 & 1.315 & 6.321 & \\
\hline Divorced/Separated & 1.059 & .400 & .240 & 1.349 & .819 & 2.221 & \\
\hline \multicolumn{8}{|l|}{ Medical Conditions } \\
\hline \multicolumn{8}{|l|}{ Nervous Disorder No } \\
\hline Nervous Disorder Yes & .550 & .208 & .008 & 1.732 & 1.153 & 2.604 & \\
\hline \multicolumn{8}{|l|}{ Mental Health No } \\
\hline Mental Health Yes & 1.380 & .415 & .001 & 3.976 & 1.762 & 8.973 & \\
\hline Hypertension & .145 & .168 & .387 & 1.156 & .832 & 1.608 & \\
\hline Psychiatric Medication & .496 & .517 & .337 & 1.643 & .596 & 4.527 & \\
\hline
\end{tabular}

Note: the dependent variable is the dichotomized PHQ-9 (mild and lower VS moderate to severe)

aggravate their mental health conditions and general well-being. Finally, comorbidities between cognitive disorders and depression may be more pronounced and severe among this age group, which may impact their well-being and overall functionality [51].

In terms of gender differences, previous research consistently reported higher prevalence rates of depression among women compared to men across studies among the general population, and this is also true of research among Syrian refugees $[13,17]$ congruent with our findings. Although some of the same justifications that have been previously cited in the literature may still be used to explain these gender-based variations, other explanations that are specific to Syrian refugees in Lebanon should be considered. For example, women in refugee settings tend to be subjected to early and forced marriage and bear family responsibilities at an early age, in addition to being exposed to sexual harassment and violence in the household and community at large [46], all of which are potential stressors that increase the risk of developing moderate to severe depression symptoms. Also, these women allude to child rearing and associated responsibilities as important sources of stress and anxiety, especially when considering their worries about the discrimination and bullying their children may face in schools in Lebanon [46].

The association between marital status and depression has been previously examined among Syrian refugees, whereby being married was found to be protective against depression in one study, potentially due to its association with social support [17], and where no relationship was found in others $[12,13,20]$. In our study, being widowed increased the odds of having depression, but this was not the case for being single or divorced. The experience of going through a death of a spouse in this community could place individuals at higher risk of developing depression than if they were single or divorced.

On the other hand, several variables were found not to be related to depression, most importantly the location of the informal tented settlement and the period of stay in Lebanon. It is possible that the properties of the four examined informal tented settlements for refugees in Lebanon entail similar living conditions that are below the appropriate standards and share similarity in terms of the availability of support and healthcare services, which translate into poor mental health among their residents. As for the period of stay in Lebanon, although 
previous studies suggested that a longer period of stay is associated with higher risk of depression [17], in our study we did not find any such association. This suggests that the period of stay in Lebanon is not correlated with the risk of developing depression, given that the mental health of Syrian refugees may be equally compromised among newcomers and long-term residents. That said, arriving in Lebanon before and after the breakout of the Syrian war in 2011 was accounted for in the study analysis, and no significant differences in depression scores were observed.

In terms of the clinical characteristics, we found that reporting a neurological condition or a history of mental illness increased the risk of depression, presumably because mental illness is directly related to depression symptoms, and neurological conditions are frequently comorbid with depression [52]. On the other hand, diabetes, CVDs and coronary artery diseases were not found to be risk factors for depression, although these have been associated with depression in previous studies $[53,54]$. Similarly, although reporting hypertension and the use of psychiatric medication have shown a statistically significant association with depression at the bivariate level of analysis, this was not replicated at the multivariable level. This means that unlike neurological conditions, non-communicable diseases may not be significant risk factors for developing depression among Syrian refugees in Lebanon. Possibly, these associations might be clouded by the overall high symptoms of depression in the study population.

In order to gain a clearer understanding of mental health disorders in this population, we recommend for future research to explore a wider range of disorders in this population such as anxiety disorders, PTSD, and substance use disorders for example to better understand their prevalence and correlates. Also, large-scale studies are needed to examine the relationship between pre and post migration factors such as legal status, living conditions, and available aids and services on the mental health of this population in this area.

\section{Recommendations \& implications}

Our findings indicate that the prevalence of depression symptoms among Syrian refugees in Lebanon is high. In light of this, an increase in screening efforts and referral mechanisms to PHCs and other health facilities is highly needed to improve access to mental health services and to reduce depression symptoms. One recommended way to do that is through task shifting, by conducting more capacity building initiatives that train non-specialized community health workers to deliver basic mental health services to their communities and to conduct referrals $[55,56]$. According to a recent national facility assessment, almost $32 \%$ of PHCs in Lebanon are currently delivering mental health services following the mhGAP training, and almost $50 \%$ of them cover rural areas [57], where most refugees reside. The integration of telemental health services into PHCs, community-based organizations, and other healthcare facilities that could be accessed by refugees may also be a suitable option to enhance the reach of these services [58, 59]. Telemental health services have strong potential in overcoming traditional barriers to mental health service use such as transportation challenges, unequal distribution of specialists among others, and may enhance access to services [58]. Importantly, considering the mobility restrictions brought forth by the COVID-19, telemental health adheres to the social distancing requirements, and enables access to mental health services for remote and underserved populations [45]. PHCs in Lebanon are usually equipped with the minimally required technological hardware and software needed for that, they tend to have minimally available specialized mental health care services, and their catchment areas tend to reach rural and underserved populations [57]. To that end, special attention should be given to high-risk groups such as older adults, women, and widowed individuals, particularly those with a history of neurological or mental health conditions. More importantly, using a culturally-sensitive approach, efforts to combat social stigma and improve mental health literacy in these communities are necessary to encourage treatment seeking when available. For example, one recent qualitative study suggested that collaborations between mental health professionals and community religious healers may be a key factor to creating pathways for referrals to mental health services [48]. Additionally, it is also important to provide minimally required and essential resources for survival, in parallel as a complement to the existing mental health services. In this regard, different aids that improve the living conditions and the quality of life for Syrian refugees are crucial, especially those that have been consistently identified as key protective factors. For example, providing options to 1) improve the financial situation in order for them to be able to meet their basic survival needs, 2) facilitate interactions with host communities and reduce tensions, and 3) improve the legal status of refugees, which help them benefit from the available services.

\section{Limitations}

Some limitations should be considered in light of our findings. First, it is important to note that the study population is not entirely homogeneous because it included individuals who came before and after the breakout of the Syrian war in 2011, which implies different levels of exposure to war trauma. Nevertheless, we accounted for this in our analysis by comparing 
depression symptoms between both groups whereby no significant differences in symptom severity were observed. Second, biases such as social desirability may have influenced the collected data (e.g. symptoms of depression, alcohol use, employment etc.) since participants were administered the questionnaire through inperson interviews rather than by self-reported means, given the high levels of reported illiteracy in informal tented settlements. Third, pre-and post- migration data such as legal status, household and living conditions, and the quality of healthcare provision among others were not collected, which could otherwise have provided a clearer association between migration status and depression. Lastly, reliance on self-reported data rather than official diagnostic reports may have resulted in under- or overestimation of the reported health conditions.

\section{Conclusion}

Mental health is of growing importance in refugee populations due to the increased vulnerability of these populations to mental health disorders. Our study findings revealed high prevalence of depression symptoms among the study population, with one in four refugees meeting criteria for moderate to severe depression symptoms. Furthermore, we found an association between potential risk factors such as, being of older age, a woman, widowed, having a neurological condition or a history of mental illness and the increased odds of having depression symptoms. Our findings bear important public health and clinical implications on refugee health, and call for the enhancement of screening efforts, the need to improve access and referral to mental health services, and the importance of improving post-migration factors such as those related to living conditions, acculturation, and legal status.

\section{Supplementary Information}

The online version contains supplementary material available at https://doi. org/10.1186/s12889-021-10266-1.

Additional file 1. This questionnaire showcases the variables used to collect data through the Sijilli Database.

\section{Abbreviations \\ AUB: American University of Beirut; COVID-19: Corona virus diseases - 19; CVD: Cardiovascular disease; GHI: Global Health Institute; IRB: Institutional Review Board; MDD: Major Depression Disorder; mhGAP: Mental Health Gap Action Program; MHPSS-TF: Mental Health and Psycho Social Support - Task Force; MoPH: Ministry of Public Health; NMHP: National Mental Health Program; OBGYN: Obstetrics and Gynaecology; PHC: Primary Healthcare Center; PHQ-9: Patient Health Questionnaire - 9; PTSD: Post traumatic stress disorder; WHO: World Health Organization}

\section{Acknowledgements}

We wish to thank the following NGOs that facilitated access to the refugees across Lebanon to complete data collection of the Sijilli EHR: Malaak, Makhzoumi Foundation, and Beyond Association. We wish to also thank the volunteer health professionals who were involved in data collection including Dr. Lara Nahouli, Dr. Ghaidaa El Saddik, Mr. Mohamad Jamal Obeid, Mr. Abdul Ghani Abou Koura, Mr. Mohamad Najdi, and Mr. Nader Husseini.

\section{Availability of the data and materials}

The data can be made available following reasonable request to the corresponding author.

\section{Authors' contributions}

The study was designed and conceptualized by HN, NEA, DN, and SS. HN wrote the first draft, and contributed to data analysis and final revisions. NEA coordinated the overall project, contributed to writing the methodology, and edited the manuscript. DN contributed to data analysis, review, and editing of the manuscript. LA performed revisions and editing of the manuscript. HD conducted full data analysis, wrote parts of the methodology section, and edited the manuscript. RH provided support for data management and data analysis and edited the draft. SS reviewed and provided critical comments on the manuscript. All authors reviewed and approved the final version.

\section{Funding}

This research did not receive any financial support.

\section{Ethics approval and consent to participate}

The present study was approved by the Institutional Review Board at the American University of Beirut. Informed consent was received by all subjects in written form when they were enrolled.

\section{Consent for publication}

Not applicable.

\section{Competing interests}

The authors declare that they have no competing interests.

\section{Author details \\ ${ }^{1}$ Global Health Institute, American University of Beirut, Beirut, Lebanon. ${ }^{2}$ School of Pharmacy, Lebanese American University, Beirut, Lebanon. \\ ${ }^{3}$ Faculty of Health Sciences, American University of Beirut, Beirut, Lebanon.}

Received: 18 August 2020 Accepted: 19 January 2021

Published online: 26 January 2021

\section{References}

1. Hassan G, Ventevogel P, Jefee-Bahloul H, Barkil-Oteo A, Kirmayer LJ. Mental health and psychosocial wellbeing of Syrians affected by armed conflict. Epidemiol Psychiatr Sci. 2016;25(2):129-41.

2. Almoshmosh N, Jefee Bahloul H, Barkil-Oteo A, Hassan G, Kirmayer L. Mental health of resettled Syrian refugees: a practical cross-cultural guide for practitioners. J Ment Health Train Educ Pract. 2019;15(1):20-32.

3. UNHCR. Syria regional refugee response: United Nations High Commissionner for Human Rights; 2018.

4. Refaat M, Mohanna K. Syrian refugees in Lebanon: facts and solutions Lancet. 2013;382:763-4.

5. Knudsen A. Syria's refugees in Lebanon: brothers, burden, and bone of contention. In: Lebanon facing the Arab uprisings. London: Palgrave Pivot; 2016. p. 135-54

6. El Chammay R, Ammar W. Syrian crisis and mental health system reform in Lebanon. Lancet. 2014;384(9942):494.

7. Karam E, El Chammay R, Richa S, Naja W, Fayyad J, Ammar W. Lebanon: mental health system reform and the Syrian crisis. Br J Psychiatry. 2016;13(4): $87-9$.

8. Jefee-Bahloul H, Moustafa MK, Shebl FM, Barkil-Oteo A. Pilot Assessment and Survey of Syrian Refugees' Psychological Stress and Openness to Referral for Telepsychiatry (PASSPORT study). Telemed e-Health. 2014;20(10): 977-9.

9. Abou-Saleh MT, Hughes P. Mental health of Syrian refugees: looking backwards and forwards. Lancet Psychiatry. 2015;2(10):870-1.

10. Weine S. Developing preventive mental health interventions for refugee families in resettlement. Fam Process. 2011;50:410-30. 
11. El Arnaout N, Rutherford S, Zreik T, Nabulsi D, Yassin N, Saleh S. Assessment of the health needs of Syrian refugees in Lebanon and Syria's neighboring countries. Confl Heal. 2019;13(1):1-14.

12. Acarturk C, Cetinkaya M, Senay I, Gulen B, Aker T, Hinton D. Prevalence and predictors of posttraumatic stress and depression symptoms among Syrian refugees in a refugee camp. J Nerv Ment Dis. 2018;206(1):40-5.

13. Georgiadou E, Zbidat A, Schmitt G, Erim Y. Prevalence of mental distress among Syrian refugees with residence permission in Germany: a registrybased study. Front Psychiatry. 2018;9(393):1-12.

14. IHME. Global burden of disease compare: Institute for Health Metrics and Evaluation; 2020.

15. May M, Klonsky D. What distinguishes suicide attempters from suicide ideators? A meta-analysis of potential factors. Clin Psychol Sci Pract. 2016;23: 5-20.

16. Rogers ML, Ringer FB, Joiner TE. The association between suicidal ideation and lifetime suicide attempts is strongest at low levels of depression. Psychiatry Res. 2018;270(August):324-8 Available from: https://doi.org/10. 1016/j.psychres.2018.09.061.

17. Poole DN, Hedt-Gauthier B, Liao S, Raymond NA, Bärnighausen T. Major depressive disorder prevalence and risk factors among Syrian asylum seekers in Greece. BMC Public Health. 2018;18(1):1-9.

18. Ventevogel P, Van O, Schilperoord M, Saxena S. Improving mental health care in humanitarian emergencies. Bull World Health Organ. 2015;93(10):666

19. Lindert J, Ehrenstein OS V, Priebe S, Mielck A, Brähler E. Depression and anxiety in labor migrants and refugees - a systematic review and metaanalysis. Soc Sci Med. 2009;69(2):246-57.

20. Naja WJ, Aoun MP, El Khoury EL, Abdallah FJB, Haddad RS. Prevalence of depression in Syrian refugees and the influence of religiosity. Compr Psychiatry. 2016;68:78-85 Available from: https://doi.org/10.1016/j. comppsych.2016.04.002.

21. Doocy S, Sirois A, Tileva M, Storey JD, Burnham G. Chronic disease and disability among Iraqi populations displaced in Jordan and Syria. Int J Health Plann Manag. 2013;28(1):1-12.

22. Llosa AE, Ghantous Z, Souza R, Forgione F, Bastin P, Jones A, et al. Mental disorders, disability and treatment gap in a protracted refugee setting. $\mathrm{Br} J$ Psychiatry. 2014;204(3):208-13.

23. Jefee-Bahloul H, Barkil-Oteo A, Pless-Mulloli T, Fouad FM. Mental health in the Syrian crisis: beyond immediate relief. Lancet. 2015;386(10003):1531 Available from: https://doi.org/10.1016/S0140-6736(15)00482-1.

24. Taher R, Allan T. Posttraumatic growth in displaced Syrians in the UK: a mixed-methods approach. J Loss Trauma. 2020;25(4):333-47 Available from: https://doi.org/10.1080/15325024.2019.1688022.

25. Porter M, Haslam N. Predisplacement and postdisplacement of refugees and internally displaced persons. J Am Med Assoc. 2005;294(5):610-2 Available from: http://jama.jamanetwork.com/article.aspx?articleid=201335.

26. Kerbage H, Marranconi F, Chamoun Y, Brunet A, Richa S, Zaman S. Mental health services for Syrian Refugees in Lebanon: perceptions and experiences of professionals and refugees. Qual Health Res. 2020;30(6):849-64.

27. Blanchet K, Fouad FM, Pherali T. Syrian refugees in Lebanon: the search for universal health coverage Mr Ruwan Ratnayake. Confl Heal. 2016;10(1):1-5 Available from: https://doi.org/10.1186/s13031-016-0079-4.

28. Jayawickreme N, Mootoo C, Fountain C, Rasmussen A, Jayawickreme E, Bertuccio R. Post-conflict struggles as networks of problems: a network analysis of trauma, daily stressors and psychological distress among Sri Lankan war survivors. Soc Sci Med. 2017;190:119-32.

29. Killikelly C, Bauer S, Maercker A. The assessment of grief in refugees and post-conflict survivors: A narrative review of etic and emic research. Front Psychol. 2018;9(OCT):1-12.

30. UNHCR. Lebanon crisis response plan. Beirut; 2019

31. Mourtada R, Schlecht J, Dejong J. A qualitative study exploring child marriage practices among Syrian conflict-affected populations in Lebanon. Confl Heal. 2017;11(Suppl 1).

32. $\operatorname{Sim}$ A, Bowes L, Gardner F. The promotive effects of social support for parental resilience in a refugee context: a cross-sectional study with Syrian mothers in Lebanon. Prev Sci. 2019;20(5):674-83.

33. El Chammay R, Karam E, Ammar W. Mental health reform in Lebanon and the Syrian crisis. Lancet Psychiatry. 2016;3(3):202-3 Available from: https:// doi.org/10.1016/S2215-0366(16)00055-9.

34. El Chammay R, Kheir W, Alaouie H. Assessment of mental health and psychosocial support services for Syrian refugees in Lebanon. Unhcr. 2013; (December):1-68.
35. MoPH. Mental health and substance use - prevention, promotion, and treatment: situation analysis and strategy for Lebanon 2015-2020 Version 1. 1: Beirut, Lebanon; 2015.

36. Morina N, Akhtar A, Barth J, Schnyder U. Psychiatric disorders in refugees and internally displaced persons after forced displacement: A systematic review. Front Psychiatry. 2018;9(SEP).

37. Saleh S, El Arnaout N, Faulkner JR, Sayegh MH. Sijilli: a mobile electronic health records system for refugees in low-resource settings. Lancet Glob Health 2019;7(9):e1168-9. Available from: https://doi.org/10.1016/S2214109X(19)30334-1

38. UNHCR. Operational portal: refugee situations. 2020.

39. Poole DN, Liao S, Larson E, Hedt-Gauthier B, Raymond NA, Bärnighausen T, et al. Sequential screening for depression in humanitarian emergencies: a validation study of the patient health questionnaire among Syrian refugees. Ann General Psychiatry. 2020;19(1):1-10 Available from: https://doi.org/10. 1186/s12991-020-0259-x

40. Kroenke K, Spitzer R, Williams J. The PHQ-9: validity of a brief depression severity measure. J Gen Intern Med. 2001;16:606-13.

41. Sawaya H, Atoui M, Hamadeh A, Zeinoun P, Nahas Z. Adaptation and initial validation of the Patient Health Questionnaire - 9 (PHQ-9) and the Generalized Anxiety Disorder - 7 Questionnaire (GAD-7) in an Arabic speaking Lebanese psychiatric outpatient sample. Psychiatry Res. 2016;239: 245-52 Available from: https://doi.org/10.1016/j.psychres.2016.03.030.

42. Karam EG, Mneimneh ZN, Dimassi H, Fayyad JA, Karam AN, Nasser SC, et al. Lifetime prevalence of mental disorders in Lebanon: first onset, treatment, and exposure to war. PLoS Med. 2008;5(4):0579-86.

43. Youssef J. Economic overview Lebanon. Lebanon: Beirut; 2020.

44. Tharoor I. Beirut's blast and Lebanon's deeper crisis. Wash Post. 2020.

45. Whaibeh $\mathrm{E}$, Mahmoud $\mathrm{H}, \mathrm{Naal} \mathrm{H}$. Telemental health in the context of a pandemic: the COVID-19 experience. Curr Treat Options Psychiatry. 2020.

46. Noubani A, Diaconu K, Ghandour L, El Koussa M, Loffreda G, Saleh S. A community-based system dynamics approach for understanding factors affecting mental health and health seeking behaviors in Beirut and Beqaa regions of Lebanon. Glob Health. 2020;16(1):1-13.

47. Masri S, Srour I. Assessment of the impact of syrian refugees in lebanon and their employment profile: International Labour Organization; 2014.

48. Al Laham D, Ali E, Mousally K, Nahas N, Alameddine A, Venables E. Perceptions and health-seeking behaviour for mental illness among Syrian refugees and Lebanese community members in Wadi Khaled, North Lebanon: a qualitative study. Community Ment Health J. 2020;56(5):875-84 Available from: https://doi.org/10.1007/s10597-020-00551-5.

49. Chemali Z, CPC B, Johnson K, Khair S, Fricchione GL. Needs assessment with elder Syrian refugees in Lebanon: Implications for services and interventions, vol. 13: Global Public Health; 2018. p. 1216-28.

50. Bazzi L, Chemali Z. A conceptual framework of displaced elderly Syrian refugees in Lebanon: challenges and opportunities. Global J Health Sci. 2016;8(11):54

51. Adams KB, Moon H. Subthreshold depression: characteristics and risk factors among vulnerable elders. Aging Ment Health. 2009;13(5):682-92.

52. Bulloch AGM, Fiest KM, Williams JVA, Lavorato DH, Berzins SA, Jetté N, et al. Depression - a common disorder across a broad spectrum of neurological conditions: a cross-sectional nationally representative survey. Gen Hosp Psychiatry. 2015;37(6):507-12 Available from: https://doi.org/10.1016/j. genhosppsych.2015.06.007

53. Elderon L, Whooley MA. Depression and cardiovascular disease. Prog Cardiovasc Dis. 2013;55(6):511-23 Available from: https://doi.org/10.1016/j. pcad.2013.03.010.

54. Mezuk B, Eaton WW, Albrecht S, Golden SH. Depression and type 2 diabetes over the lifespan: a meta-analysis. Diabetes Care. 2008;31(12):2383-90.

55. Naal H, El Koussa M, El Hamouch M, Hneiny L, Saleh S. A systematic review of global health capacity building initiatives in low-to middle-income countries in the Middle East and North Africa region. Glob Health. 2020;16(1).

56. Hoeft TJ, Fortney JC, Patel V, Unützer J. Task-sharing approaches to improve mental health care in rural and other low-resource settings: a systematic review. J Rural Health. 2018;34(1):48-62.

57. Hemadeh R, Kdouh O, Hammoud R, Jaber T, Khalek L. The primary health care network in Lebanon: a national facility assessment Randa. East Mediterr Health J. 2020:1-9 Available from: https://www.golder.com/insights/blockcaving-a-viable-alternative/.

58. Naal H, Whaibeh E, Mahmoud H. Guidelines for primary health care-based telemental health in a low-to middle-income country : the case of Lebanon. 
Int Rev Psychiatry. 2020;0(0):1-9 Available from: https://doi.org/10.1080/ 09540261.2020 .1766867$.

59. Almoshmosh N, Jefee-Bahloul H, Abdal-lah W, Barkil-Oteo A. Use of storeand-forward tele-mental health for displaced Syrians. Intervention. 2020; 18(1):66-70

\section{Publisher's Note}

Springer Nature remains neutral with regard to jurisdictional claims in published maps and institutional affiliations.

- fast, convenient online submission

- thorough peer review by experienced researchers in your field

- rapid publication on acceptance

- support for research data, including large and complex data types

- gold Open Access which fosters wider collaboration and increased citations

- maximum visibility for your research: over $100 \mathrm{M}$ website views per year

At BMC, research is always in progress. 